\title{
Criminologie
}

\section{Pour une approche intégrative de la conduite délinquante des adolescents}

\section{Marc LeBlanc}

Volume 19, numéro 1, 1986

Politiques et pratiques pénales. 25 ans de réflexion et d'action

URI : https://id.erudit.org/iderudit/017227ar

DOI : https://doi.org/10.7202/017227ar

Aller au sommaire du numéro

Éditeur(s)

Les Presses de l'Université de Montréal

ISSN

0316-0041 (imprimé)

1492-1367 (numérique)

Découvrir la revue

Citer cet article

LeBlanc, M. (1986). Pour une approche intégrative de la conduite délinquante des adolescents. Criminologie, 19(1), 73-95. https://doi.org/10.7202/017227ar
Résumé de l'article

During the I960's, the founders of criminology wanted to have it recognized as an autonomous discipline, not only because of its subject matter, but above all by assigning it an integrative mission. In this article, we propose to take this kind of integrative approach vis-à-vis the delinquent behaviour of adolescents. Based on a precise knowledge of the major forms of delinquent conduct, we formulate and validate a multidisciplinary theory of its emergence and development. With the aid of a typology of the delinquent, we then focus our attention on the practical implications of this knowledge of the subject and of the discovery of the mechanisms of its etiology. In this way, criminology can be shown to be both a science and multidisciplinary profession, hence integrative. 


\section{POUR UNE APPROCHE INTÉGRATIVE DE LA CONDUITE DÉLINQUANTE DES ADOLESCENTS *}

Marc LeBlanc**

During the 1960's, the founders of criminology wanted to have it recognized as an autonomous discipline, not only because of its subject matter, but above all by assigning it an integrative mission. In this article, we propose to take this kind of integrative approach vis-à-vis the delinquent behaviour of adolescents. Based on a precise knowledge of the major forms of delinquent conduct, we formulate and validate a multidisciplinary theory of its emergence and development. With the aid of a typology of the delinquent, we then focus our attention on the practical implications of this knowledge of the subject and of the discovery of the mechanisms of its etiology. In this way, criminology can be shown to be both $a$ science and multidisciplinary profession, hence integrative.

\section{À L'OCCASION D'UN ANNIVERSAIRE}

1960-1985 : l'enseignement de la criminologie se poursuit depuis vingt-cinq années à l'Université de Montréal. Cet anniversaire commande une introduction personnelle à cet article sur l'approche intégrative, ceci d'autant plus que l'auteur a été présent dans cette unité d'enseignement au cours des vingt dernières années de cette expérience unique. Il ne saurait être question que nous proposions un bilan des progrès et déboires de l'approche intégrative au cours de cette période, plutôt nous jetterons les jalons pour une évaluation de l'atteinte des buts que nous présentaient les pionniers de la criminologie moderne.

En 1965, un des premiers sujets de réflexion qui était proposé aux étudiants de maîtrise découlait de la question suivante: comment définir la criminologie? Les définisseurs de la «nouvelle»

- Cette recherche a été subventionnée par le Conseil de recherche en sciences humaines du Canada et l'Université de Montréal.

** Professeur titulaire à l'École de criminologie de l'Université de Montréal, chercheur associé au Centre international de criminologie comparée. 
criminologie, plus particulièrement Szabo et Wolfgang, affirmaient alors que son objet, le crime, la criminalité et le criminel, ne pouvait lui servir d'unique raison d'être. La criminologie, pour exister comme discipline, se devait d'être une science et une profession muldisciplinaire. Une science, c'est-à-dire une discipline qui développe les connaissances à partir d'un va et vient continuel entre la réflexion théorique et la recherche empirique. Une profession, c'est-à-dire une praxis qui articule les connaissances et l'action, tant au niveau des politiques criminelles que de l'intervention directe auprès des individus et des groupes. L'une et l'autre fondues au creuset de la multidisciplinarité. Cette conception novatrice trouve son apogée dans le XVII ${ }^{e}$ Cours international de criminologie qui a eu lieu à Montréal en 1967 (Szabo, 1968).

Cette définition de la criminologie nous proposait une mission spécifique : réaliser des intégrations. Mission que nous avons fait nôtre et qui nous enjoignait de rassembler les notions et données provenant de diverses disciplines, de développer des interprétations à la lumière de faits issus des recherches empiriques et de dégager les implications des connaissances scientifiques pour la pratique criminologique. Cette définition novatrice de la criminologie nous soumettait un programme emballant: tenter une approche intégrative du phénomène criminel. Approche compréhensive que peu de disciplines scientifiques et de professions proposent comme idéal et qui demeure encore rarement pratiquée dans les écrits criminologiques du dernier quart de siècle.

Au cours des quinze dernières années nous nous sommes naïvement attaqués à cette tâche colossale en nous servant de deux moyens : la recherche et la consultation. Avec l'aide de collègues et de nombreux assistants, dont nous ne saurions passer sous silence l'indispensable contribution au cours de ces années, nous avons mené des recherches empiriques qui nous ont permis de caractériser la conduite et la carrière délinquante, de développer et de valider un modèle explicatif de son émergence et de son développement et d'élucider les mécanismes de la réaction sociale formelle. En somme, de proposer des intégrations entre théories et données empiriques et entre notions et données provenant de cinq sources : la sociologie, la psychologie, le droit, l'application de la loi et le phénomène délinquant lui-même.

Parallèlement, nos activités de consultation auprès de divers organismes ont exigé que nous portions une attention particulière 
aux implications des résultats de nos recherches empiriques et de nos formulations théoriques. Ainsi nous avons esquissé des tentatives d'intégration des connaissances scientifiques et de la pratique, tant au niveau des politiques criminelles que de l'intervention directe auprès des délinquants, ceci, entre autres, par l'intermédiaire de la construction d'une typologie multidimensionnelle.

Cet article veut refléter l'approche intégrative que nous avons adoptée face à la délinquance des mineurs. Cette illustration rapide d'une tentative d'intégration, somme toute encore limitée, permettra peut-être aux lecteurs de répondre à trois questions essentielles : l'approche intégrative, que les pionniers nous proposait pour marquer la spécificité de la criminologie, est-elle toujours pertinente? Dans quelle mesure la criminologie d'aujourd'hui, à Montréal ou ailleurs, s'en approche? avec quel degré de succès l'intégration proposée dans cet article réalise-t-elle la mission que nous avons consciemment acceptée?

En espérant que chaque réponse que vous donnerez à ces questions sera une source de réflexions personnelles et collectives fructueuses sur la définition de la criminologie actuelle et sur les orientations à lui donner dans l'avenir, nous soumettons donc humblement l'état actuel de notre intégration à titre illustratif.

\section{LES FORMES DE LA CONDUITE DÉLINQUANTE}

La pierre angulaire de la définition de la criminologie, c'est son objet; la conduite délinquante devient nécessairement la variable clé de toute démarche criminologique. Par ailleurs, les définitions légales, les réflexions théoriques et les recherches empiriques vont généralement plus loin et elles acceptent de plus en plus une définition restreinte de la délinquance : les infractions criminelles. Le développement des connaissances peut aussi se rentabiliser car les chercheurs maximisent la spécificité, la constance et l'homogénéité de l'objet d'étude et de mesure. De plus, la criminologie optimalise sa signification parce qu'elle s'élabore à partir de comportements hautement visibles socialement, et même stables dans l'espace et le temps, et elle s'exprime aussi sur le même registre que celui des instances de régulation sociale.

Ce retour à la problématique fondamentale de la criminologie ne nie pas la pertinence qu'il y a à étudier la déviance, il marque le besoin d'une science appliquée qui supporte une pratique professionnelle. Cette convergence du droit pénal et de la crimi- 
nologie des mineurs ne saurait masquer certains problèmes de délimitation du clavier de la délinquance. Il est exact que le Code criminel compte encore des conduites problématiques au sens qu'il ne s'agit pas d'infractions contre les personnes et les biens; toutefois, il ne s'agit pas des infractions les plus fréquemment réprimées, ni des questions que nous introduisons dans nos instruments de mesure de la délinquance cachée. Les infractions criminelles, une fois devenues l'objet principal de la criminologie des mineurs, constituent une base commune de travail pour les législateurs, gestionnaires, praticiens et chercheurs.

Nos travaux sur la délinquance cachée des adolescents et pupilles du tribunal (Fréchette et LeBlanc, 1979, 1985) montrent qu'il faut retenir que la caractéristique fondamentale du phénomène de la délinquance, c'est qu'elle se révèle comme une activité avant tout adolescente, nous avons même parlé d'un épiphénomène. Toutefois, il ne faut pas oublier qu'elle s'inscrit comme un phénomène social inquiétant puisque certaines de ses manifestations se distinguent par une fréquence d'occurrence élevée, par de la gravité objective et par de la précocité. Par ailleurs, cette conduite demeure fluide, elle est appelée à s'intensifier rapidement et aussi à décliner brusquement; c'est souvent un épisode. Cette convergence de l'intensité et de l'imprévisibilité, comme traits dominants de la délinquance des adolescents, rend impérieuse la nécessité de distinguer ses modes d'expressions spécifiques.

Nous en avons identifié trois : la délinquance d'occasion, la délinquance de transition et la délinquance de condition. La première forme, la délinquance d'occasion, affecte plus de $80 \%$ des adolescents et elle se manifeste brièvement par des délits mineurs; ce caractère bénin, voire même insignifiant, nous la font interpréter comme faisant partie des risques ordinaires de la vie, c'est le prix que doit payer un adolescent pour s'adapter à une vie sociale complexe, pour apprendre les normes et les frontières des conduites socialement tolérées.

Le second mode, la délinquance de transition, touche moins de $10 \%$ des adolescents et autour de la moitié des pupilles du tribunal. Elle s'expose comme étant assez substantielle, hétérogène et parfois parsemée de délits plus graves, mais limitée à une période circonscrite dans le temps; nous la comprenons comme une poussée délictueuse liée à des difficultés particulières de la vie des sujets : des conflits familiaux et/ou scolaires au début de 
l'adolescence, des problèmes de transition statutaire à la fin de cette période et/ou au début de l'âge adulte.

La troisième configuration, la délinquance de condition, se retrouve chez moins de $1 \%$ des adolescents et chez environ les deux cinquièmes des pupilles du tribunal que nous avons étudiés. Elle est une conduite stabilisée dans l'activité du sujet; elle est intégrée parce que précoce, volumineuse, hétérogène, aggravée et persistante même à l'âge adulte. L'ampleur de l'activité délictueuse est le cœur d'un véritable fonctionnement antisocial, d'un mode de vie, d'une carrière délinquante.

Il est évident que toutes les instances de notre société, les parents, les éducateurs, les policiers, les juges, les intervenants sociaux, les internats, ... ne peuvent réagir de façon identique à ces trois styles de délinquance. Les politiques sociales de prévention, de répression et de traitement de la délinquance ne sauraient s'appuyer sur les mêmes principes, ni s'appliquer avec la même rigueur face à ces trois types de délinquance. Les praticiens, dans leur action clinique, ne peuvent utiliser les mêmes techniques d'intervention pour faire face à ces configurations de délinquance. Ces trois modes d'expression de la délinquance, même en ne se limitant qu'au comportement, appellent une stratégie différentielle d'action face à la délinquance des mineurs.

En conséquence, l'ensemble de nos données sur la conduite délinquante des mineurs pointent vers la nécessité de dédramatiser la majeure partie de leurs activités délinquantes et, comme une grande tolérance existe déjà, vers la nécessité impérative de maintenir celle-ci face aux délinquances d'occasion et de transition. On peut accepter ce principe premier d'une politique sociale à l'égard de la délinquance des mineurs sans crainte; en effet, nos données (Fréchette et LeBlanc, 1985) établissent aussi que les problèmes de délinquance chronique s'imposent clairement aux instances de régulation sociale dès le milieu de l'adolescence.

Par ailleurs, nos données nous indiquent aussi qu'il est nécessaire d'intervenir précocement, puissamment et intensivement face à la délinquance de condition pour empêcher que les jeunes s'enlisent dans l'antisocialité à un point tel que toute leur fonctionnalité d'adulte s'en trouve compromise. La seule conciliation entre ces deux exigences réside évidemment dans la prédiction différentielle, certains délinquants justifiant la non-intervention, 
d'autres requérant une action massive. Tout le défi de la criminologie appliquée est là.

Au Québec, les récentes législations fédérale (Loi sur les jeunes contrevenants, 1982) et provinciale (Loi de la protection de la jeunesse, 1984) constituent un cadre législatif tout à fait approprié pour le développement d'une véritable stratégie différentielle d'intervention face au phénomène de la délinquance des mineurs. Premièrement, elles spécifient la notion de délinquance et elles la limitent aux infractions au Code criminel; en soi cette position, d'une part, facilite le travail de différenciation que les décideurs doivent constamment accomplir et, d'autre part, elle rend nécessaire une distinction plus fine parmi les jeunes délinquants.

Deuxièmement ces législations favorisent l'application d'une stratégie différentielle parce qu'elles proposent un éventail plus vaste de mesures : des mesures de rechange à la judiciarisation : conciliation avec la victime, restitution, travaux communautaires, ... et une gamme plus précise de possibilités d'ordonnance : garde ouverte, garde fermée, probation, ... Par ces mesures plus spécifiques et plus variées, les législations obligent les intervenants à mieux définir la pertinence de chacune d'entre elles pour chaque type de jeunes délinquants.

C'est non seulement la variété des mesures qui encourage une stratégie différentielle, mais elle est aussi renforcée par la mécanique judiciaire, qui par la représentation légale, l'appel, ... favorise le choix de la mesure nécessaire et suffisante dans chaque cas particulier. Elle est aussi supportée par l'éventail des rôles que l'on attribue aux spécialistes des sciences humaines: ils participent à la décision concernant le triage des jeunes délinquants qui devront répondre de leurs actes devant le tribunal; ils conseillent les juges lors de la décision grâce à leurs rapports prédécisionnels; ils appliquent les mesures ordonnées; ils déterminent la fin des mesures sous réserve de l'approbation de l'autorité judiciaire. $\dot{A}$ travers toutes ces tâches, ils devront exercer une discrimination adéquate parmi les jeunes délinquants.

Le cadre administratif convient aussi très bien à une stratégie différentielle; de fait, la structure des services, qui assurent l'application de la loi, n'est pas contre-indiquée pour l'éclosion d'une approche différentielle. Ainsi, les services de police tamisent selon des critères précis de dangerosité (LeBlanc, 1971), tout 
comme les substituts du procureur général (Trépanier et Gagnon, 1984), et ils disposent, en plus, de critères inscrits dans diverses législations ou règlementations. Pour sa part, la Direction de la protection de la jeunesse ou le Directeur provincial constitue un lieu idéal pour l'évaluation et l'orientation différenciée des jeunes délinquants, car virtuellement, tous les cas y viennent avant ou après la judiciarisation. L'organisation des services sociaux n'est pas en reste (centres de services sociaux, centres locaux de services communautaires, centres d'accueil, orgagnismes communautaires, ...) ; s'il est un problème ce n'est pas celui de l'absence de variété parmi les services, c'est plutôt celui de l'absence de spécificité dans la nature de la clientèle desservie par les divers services (LeBlanc, 1983a; 1985a).

Ces commentaires sur le phénomène de la délinquance nous ont permis de proposer deux des sortes d'intégration dont nous avons traité au début de cet article. La première, celle des faits et de la théorie, se présente ainsi : à partir de nos observations sur la conduite délinquante, nous contestons la position théorique courante qui conçoit la délinquance comme un tout indifférencié, un continuum de degré, et nous proposons des configurations qualitativement différentes sans lien séquentiel automatique entre elles. La seconde, celle de la science et de la pratique, s'affirme ainsi : compte tenu de la diversité des formes de la délinquance une stratégie différentielle est nécessaire et elle doit s'exprimer en conciliant deux principes en apparence contradictoires, la tolérance face à la majeure partie de la délinquance et l'intervention précoce face à la délinquance de condition.

\section{UN PARADIGME THÉORIQUE}

Depuis que Lewin (1933) a proposé sa célèbre formule : $B=(P)$ (E) où la conduite (B) est fonction des caractéristiques de la personne (P) et de son milieu (E), les explorations ont été nombreuses pour encourager les théoriciens à développer des théories intégratives de la conduite, c'est-à-dire des théories tenant compte simultanément de facteurs sociologiques et psychologiques. En criminologie, les tentatives sont intéressantes, mais dans l'ensemble elles sont soit psychologisante (Jessor et al., 1968, 1977), soit sociologisante (Reiss, 1951; Martin et al., 1965; Szabo et al., 1968;), soit multifactorielle (West, 1982) ou elles ne rendent compte que d'un phénomène particulier, par exemple la violence (Wolfgang et Ferracuti, 1967). 
Dans le cadre de nos travaux, depuis 1975, nous avons été amenés à développer et à vérifier empiriquement une théorie intégrative de l'émergence et du développement de la conduite délinquante au cours de l'adolescence. En nous appuyant sur les formulations et vérifications initiales (Leblanc et Biron, 1981; Le Blanc, 1983b); et sur les travaux plus récents Frechette et LeBlanc, 1985; LeBlanc et al., 1985; LeBlanc et Ouimet, en cours. il devient possible de proposer une formulation plus définitive de cette théorie et de rapporter les résultats des validations réalisées jusqu'ici.

\subsection{Sa formulation}

De la manière la plus simple et la plus générale, la théorie se résume par la phrase suivante : la délinquance émerge et se développe dans la mesure d'une faiblesse du mécanisme de régulation de la conduite. De manière plus spécifique, on peut formuler ainsi cette défaillance du mécanisme de régulation : elle s'exprime par des liens ténus avec la société, des retands dans le développement du fonctionnement psychologique et une déficience de la contrainte sociale. La défaillance momentanée de l'une ou l'autre de ces composantes du mécanisme de régulation conduit directement à une délinquance d'intensité variable ou, indirectement, à travers un processus de marginalisation qui se manifeste face à l'école et par le choix de pairs deviants, et qui constitue une entrée difficile dans le rôle d'adolescent. Cette défaillance est affectée par des conditions structurelles : sexe du sujet, statut social de la famille et niveau d'intelligence du sujet. Le schéma 1 illustre cette formulation.

Nous référons les lecteurs aux travaux cités ci-dessus, ils y trouveront les définitions et opérationnalisation, de cet ensemble de concepts. Rappelons toutefois que les liens sociaux réfèrent au degré d'attachement aux personnes et d'engagement envers les institutions sociales; la contrainte sociale doit être comprise comme la contrainte interne, l'intériorisation des normes, et la contrainte externe, les pressions et sanctions du milieu; l'entrée en rôle d'adolescent se rapporte à la conformité aux exigences sociales pour ce groupe d'âge; et la nature du fonctionnement psychologique met en exergue l'égocentrisme naturel qui s'atténue progressivement en raison de l'affaiblissement de la primitivité, de la négativité, de l'insécurité de l'hyposocialité et de l'isolement interpersonnel. 
Ces éléments régulateurs, et cela est la principale contribution de notre travail, ne sont pas articulés aléatoirement. Concrètement, deux assises interagissent, les liens sociaux et la nature du fonctionnement psychologique, dont nous ne pouvons départager la priorité pour expliquer l'ensemble de la délinquance au moment de l'adolescence, ceci en raison d'une longue histoire antérieure d'interactions. Ces deux assises de la conduite délinquante déterminent le niveau de réception de l'adolescent à la contrainte sociale, aux exigences de la vie en société; ces sources de contrainte constituent une sorte de barrage aux pressions vers l'activité illicite. Parallèlement à cette dynamique du mécanisme principal de régulation de la conduite délictueuse, il peut se développer une non-conformité au rôle d'adolescent; celle-ci s'exprime par des conflits avec l'école et un rattachement à des pairs déviants. Elle est supportée par des liens insuffisamment noués, un développement psychologique incomplet et une contrainte sociale défaillante.

SCHÉMA 1

Illustration de la théorie

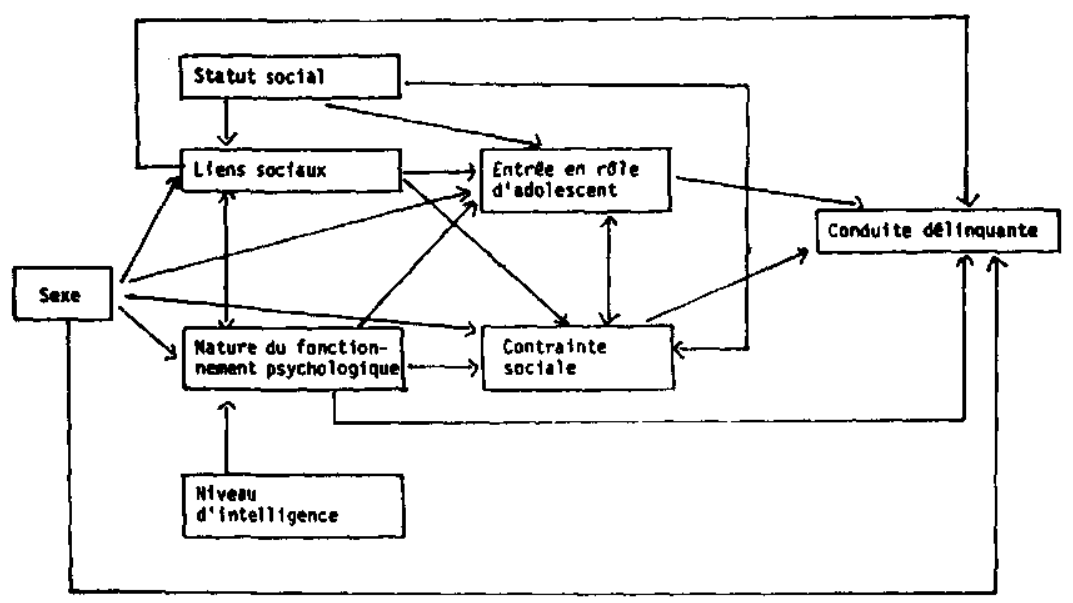

Le schéma 1 illustre aussi que deux conditions structurelles influencent divers aspects du mécanisme de régulation : le niveau d'intelligence a un impact sur la nature du fonctionnement psychologique et le statut social de la famille détermine le développement des liens sociaux, la nature de la contrainte sociale utilisée et les difficultés d'entrée en rôle d'adolescent. L'autre condition 
structurelle, le sexe, a une influence générale sur l'importance respective des composantes du mécanisme de régulation dans la production de la conduite délinquante de même qu'elle conditionne le degré de délinquance. Notons que la présence de flèches sur le schéma 1 indique les relations directes et majeures entre les composantes du mécanisme de régulation.

Cette formulation théorique propose aussi une perspective synchronique sur l'émergence et le développement de la délinquance au cours de l'adolescence. Le schéma 2 permet de visualiser cette théorie d'un point de vue diachronique. Il veut principalement rendre compte du fait que l'état antérieur des composantes du mécanisme de régulation a des influences globales et spécifiques sur la situation actuelle et, plus particulièrement, que la conduite délinquante antérieure a un impact majeur sur la conduite délinquante actuelle.

\subsection{Sa vérification}

Le paradigme théorique proposé, de nature systématique, intégratif et développemental, veut rendre compte de l'émergence et du développement de la délinquance au cours de la période de l'adolescence. Nous n'avons pu l'opérationnaliser et le vérifier intégralement sur le plan longitudinal, néanmoins, il convient de se demander dans quelle mesure sa portée est générale. Sans reprendre l'ensemble des vérifications empiriques que nous avons conduites, il convient de commenter la pertinence du modèle sur le plan transversal, son applicabilité pour les garçons et les filles et sa valeur sur le plan Iongitudinal (nous référons le lecteur à LeBlanc et Biron, 1981, et LeBlanc et Ouimet, en préparation, pour plus de détails sur l'échantillon et l'opérationnalisation des concepts).

Sur le plan transversal, il convient de conclure que les concepts retenus sont pertinents et efficaces pour rendre compte de la conduite délinquante, $52 \%$ de variance est expliquée (tableau 1); de plus, le schéma 3 montre que les liens directs hypothétisés se confirment dans l'ordre d'importance suivant: contrainte sociale, entrée en rôle d'adolescent, nature du fonctionnement psychologique, liens sociaux et sexe. L'ordonnancement général prévu est aussi reproduit par l'ensemble des corrélations : une assise, liens sociaux et fonctionnement psychologique (leurs corrélations sont plus fortes entre elles et avec les autres concepts qu'avec la conduite délinquante), influence indirectement la 
conduite délinquante à travers des conditions circonstancielles, entrée en rôle d'adolescent et contrainte sociale (leurs corrélations sont plus fortes avec la conduite délinquante qu'avec les liens et la nature du fonctionnement psychologique). Notons, au niveau des variables structurelles, des modifications à apporter au paradigme théorique : le statut social n'a un impact significatif que sur les liens sociaux.

\section{SCHÉMA 2}

Formulation développementale de la théorie

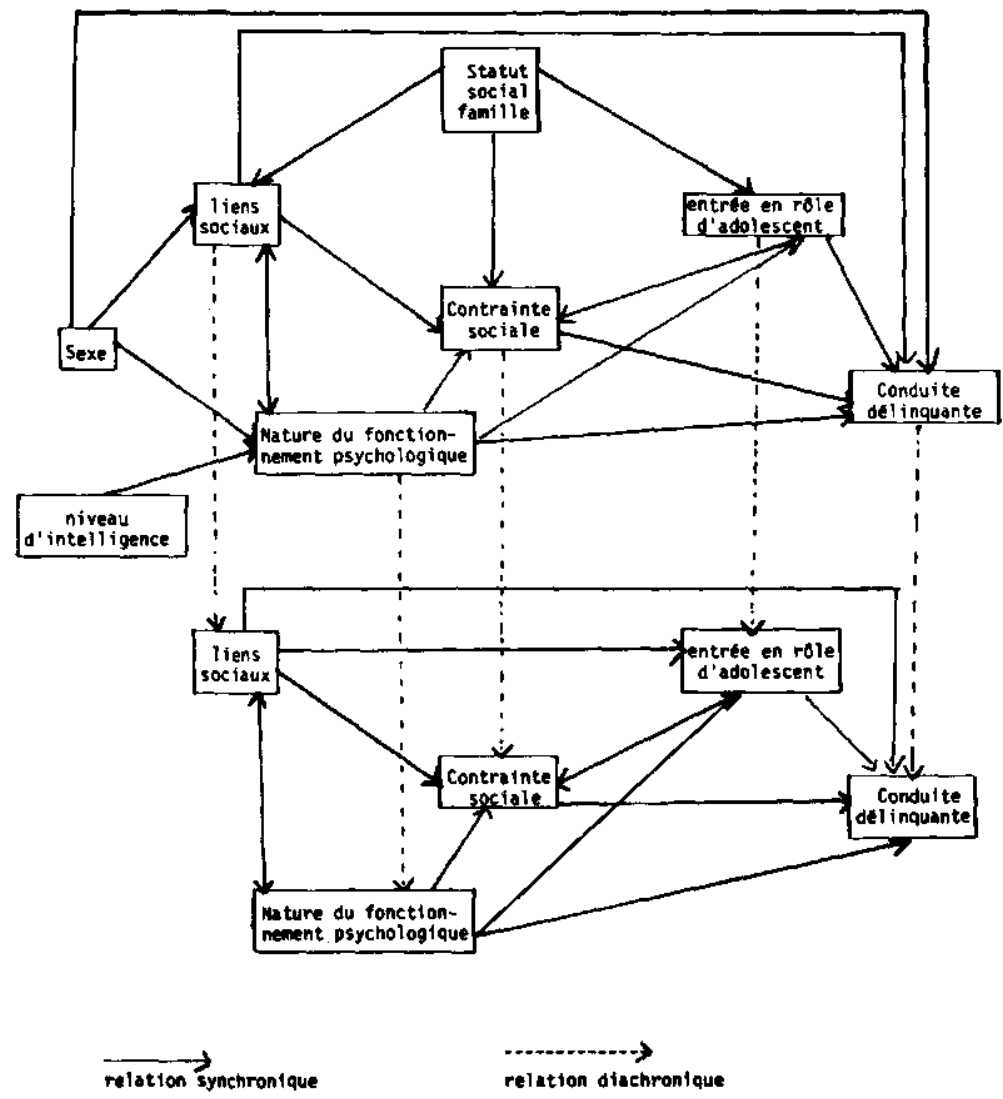

filles (voir appendice $A$ : tableaux 2 et 3 et schémas 4 et 5), En somme, les données rapportées au tableau 1 et au schéma 3 montrent la validité générale de la théorie intégrative proposée. De plus, cette théorie s'applique à la fois aux garçons et aux 
même si les concepts retenus sont moins efficaces pour ces dernières $(42 \%$ de variance expliquée pour les filles contre $55 \%$ de variance expliquée pour les garçons), car l'organisation des concepts explicatifs est la même pour les deux sexes.

\section{TABLEAU 1}

Ensemble des adolescents: intercorrélations ${ }^{1}$ et analyse de régression multiple des composantes de la théorie

\begin{tabular}{|c|c|c|c|c|c|c|}
\hline & $\begin{array}{r}\text { Sexe Statut } \\
\text { social }\end{array}$ & Liens & Contrainte & $\begin{array}{l}\text { Entrée } \\
\text { en } \\
\text { rôle }\end{array}$ & $\begin{array}{l}\text { Fonction- } \\
\text { nement } \\
\text { psycholo- } \\
\text { gique }\end{array}$ & $\begin{array}{l}\text { Conduite } \\
\text { délin- } \\
\text { quante }\end{array}$ \\
\hline $\begin{array}{l}\text { Sexe } \\
\text { Statut social } \\
\text { Liens } \\
\text { Contrainte } \\
\text { Entrée en } \\
\text { rôle } \\
\text { Fonction- } \\
\text { nement psy- } \\
\text { chologique } \\
\text { Conduite } \\
\text { délinquante }\end{array}$ & - & $\begin{array}{l}10 \\
, 22\end{array}$ & $\begin{array}{r}, 34 \\
, 12 \\
, 67\end{array}$ & $\begin{array}{l}, 20 \\
, 06 \\
, 52 \\
, 65\end{array}$ & $\begin{array}{l}, 45 \\
, 12 \\
, 62 \\
, 58 \\
, 44\end{array}$ & $\begin{array}{l}, 32 \\
, 32 \\
, 40 \\
, 66 \\
, 60 \\
, 54\end{array}$ \\
\hline
\end{tabular}

1. Il s'agit de corrélations canoniques sauf en se qui concerne les variables sexe, statut social et conduite délinquante (corrélations multiples).

Contrainte sociale

Entrée en tôle

Fonctionnement psychologique

Liens sociaux

Statut social de famille

Sexe

$\begin{array}{ccc}\mathbf{R} \text { multiple } & \mathbf{R}^{2} & \mathbf{r} \\ , 65948 & , 43491 & , 65948 \\ , 69653 & , 48516 & , 59769 \\ , 71843 & , 51613 & , 54382 \\ \mathbf{7 3 8 0 4} & , 54471 & , 40073 \\ , 73810 & , 54480 & , 04566 \\ , 73813 & , 54484 & ,, 32398\end{array}$

SCHÉMA 3

Ensemble des sujets : intercorrélations

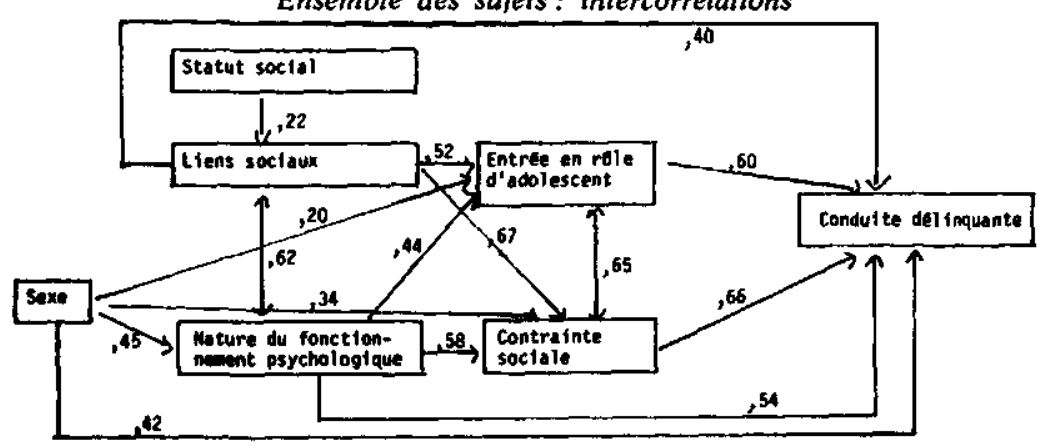


Sur le plan longitudinal, la théorie proposée augmente sa portée, car la proportion de la variance expliquée monte à $82 \%$ (appendice A, tableau 4 et schéma 6 ) et c'est la conduite délinquante au temps 1 qui est la principale responsable de la puissance explicative accrue du modèle.

Au terme de cette vérification empirique rapide, il convient de conclure que la théorie générale proposée est pertinente et valide. Pour assurer sa véritable portée et démontrer sa valeur pour guider l'intervention, il conviendra de procéder à une vérification à partir des concepts intermédiaires sous-jacents et des variables de bases qui l'opérationnalise (Leblanc et Ouimet, en préparation) .

La démarche rapportée ci-dessus montre la pertinence de réaliser deux des formes d'intégration que les pionniers affirmaient nécessaire en criminologie : l'intégration des concepts et données de diverses disciplines et l'intégration de la réflexion théorique et de la recherche empirique. Appliquons maintenant ce paradigme théorique aux formes de délinquance pour tenter une autre forme d'intégration : celle de la science et de la pratique.

\section{UNE STRATÉGIE D'INTERVENTION}

Il est possible de soutenir, à la lumière des résultats rapportés par Fréchette et LeBlanc (1985), que chaque style de délinquance est supporté par une combinaison spécifique de facteurs. Ainsi, nous avons montré que la délinquance d'occasion n'a pas sa source dans des liens sociaux inadéquats, ni dans un retard du développement psychologique, c'est plutôt une contrainte sociale relâchée, en particulier la supervision déficiente des parents, des difficultés momentanées d'adaptation scolaire et des associations avec des pairs inadaptés qui, alternativement ou en conjugaison, soutiennent l'émergence de cette délinquance.

La délinquance de transition obéit aux mêmes circonstances auxquelles peuvent s'ajouter des conflits familiaux aigus (divorce,...), mais elles sont plus lourdes. Nous avons parlé d'une délinquance de transition qui manifeste une opposition mineure au social, à travers l'école et les pairs, et qui résulte, au pire, d'un débalancement du fonctionnement psychologique à la faveur des perturbations de l'adolescence ou d'une situation de crise dans l'entourage du jeune.

Pour sa part, l'analyse comparée des facteurs criminogènes actifs du champ social démontre que les adolescents dont la 
délinquance de condition sont sous l'influence d'événements antécédents qui sont hors de leur contrôle. Ils subissent les désavantages de leur milieu en étant plus souvent originaire des milieux socio-économiques défavorisés. Ils ont accumulé des handicaps sociaux majeurs: des retards et un échec de leur adaptation scolaire; une réputation de mauvais garnement; des interventions successives et inefficaces du réseau des affaires sociales et du système de justice; ... Leur marginalisation s'est progressivement accentuée dans leur communauté immédiate et à l'école et elle s'est cristallisée par le rattachement à des pairs délinquants.

Dans un tel contexte, il n'est pas surprenant qu'ils n'aient pas développé un attachement à leurs parents ou à des personnes significatives, ni un engagement envers les institutions et les valeurs socialisées. Et il n'est pas imprévu qu'un individu, qui n'est pas plus rattaché à la société que le jeune délinquant, ne puisse pas être soumis aux contraintes sociales. En somme, les jeunes affichant ce style de délinquance apparaissent comme n'ayant pas évolué vers la socialité; ils sont en marge; ils sont dissocialisés. Ces déterminismes imposants ne nous sont pas apparus la raison d'être profonde de la délinquance de condition. C'est parmi les facteurs criminogènes du champ psychologique, notamment dans un égocentrisme qui perdure, que nous avons trouvé le fondement de cette dissocialité. À cet égard, les analyses illustrent que les dimensions psychologiques subordonnent l'influence du social : les variables de personnalité ne sont pas primordiales pour expliquer l'émergence de cette délinquance; les jeunes délinquants affichent un fonctionnement psychologique subnormal; l'intensité de leur délinquance varie de façon très satisfaisante en regard de leurs traits psychologiques; les variables qui permettent de distinguer le mieux les jeunes délinquants entre eux proviennent du champ psychologique. La dynamique qui conduit à la délinquance enracinée se manifeste à travers un égocentrisme qui se solidifie et que supporte la dissocialité.

Les délinquances d'occasion et certaines formes de délinquance de transition sont circonstancielles, elles appellent naturellement la tolérance parce que cette délinquance a moins de $10 \%$ de chance de se reproduire à l'âge adulte, elle est aléatoire (LeBlanc, 1985b). Cette forme de délinquance, par sa nature, n'est pas dépistable; on ne peut pas la prévenir, car il est impossible de prédire qui sont les adolescents qui seront soumis aux circonstances qui favorisent l'émergence de la délinquance de surface. 
La société ne peut donc faire appel qu'à une prévention primaire, une action globale sur la communauté. Dans un tel cadre de prévention, la société se doit de mettre l'accent sur la revitalisation de ses institutions : l'école, la famille, le travail et la communauté. C'est un modèle de développement social qui s'appuie sur deux stratégies : favoriser, premièrement, l'amélioration des actions éducatives habituelles des parents, de l'école et de la religion (en référence aux concepts de liens sociaux et de contrainte sociale) et, deuxièmement, mettre en place une véritable politique jeunesse qui favorise l'intégration des jeunes au travail et à la vie de la communauté (en référence au concept d'entrée en rôle d'adolescent). La plus récente formulation de ces modèles compréhensifs de prévention primaire est celle de Waller et Weiler (1984). Ils s'appuient sur la théorie de régulation dont nous avons présenté une version intégrative précédemment.

Les délinquances de transition et de condition se concrétisent suivant quatre formes principales chez les jeunes délinquants selon les travaux de Fréchette et LeBlanc $(1980,1985)$ : les délinquants marginaux, immatures, à structure névrotique et persistants graves.

Le délinquant marginal, celui dont la conduite délictueuse a été qualifiée de sporadique, s'inscrit comme un individu marqué par un certain nombre de faiblesses réelles par rapport à un adolescent conventionnel. Les déficits psychologiques accumulés et la présence de pression vers la délinquance (difficultés scolaires, pairs délinquants...) ne paraissent pas considérables, la personnalité n'est pas trop massivement affectée et surtout les liens avec autrui ne sont pas trop gravement compromis. On peut prévoir à leur égard, et prioritairement, des interventions des instances sociales et/ou judiciaires qui les garderont dans leur milieu naturel, sans dangers réels d'affecter la protection du public. Quant à la nature de l'intervention, il serait indiqué qu'elle mette l'accent sur la restauration des liens avec la communauté (en référence aux concepts de liens sociaux et de contrainte sociale); Finckenauer (1984) donne des exemples de programmes qui peuvent réduire la dissocialité de ces jeunes et qui mettent l'accent sur la réintégration sociale, principalement en agissant sur l'entourage de ces individus (réduction de l'influence des pairs inadaptés, développement d'opportunités scolaires et de travail, ...) (en référence au concept d'entrée en rôle d'adolescent).

Le délinquant immature, c'est l'adolescent dont la conduite délictueuse est persistante mais non aggravée par des délits sérieux. 
Il se singularise surtout par un rejet de l'école, par une adhésion à des schèmes de référence criminels et par un mal être objectif significatif, celui-ci s'exprime par un désengagement social et interpersonnel et de l'immaturité. Face à ce groupe, les instances judiciaires ont à intervenir compte tenu de la gravité moyenne de leurs délits (de type vol par effraction) et de leur récidive. Ce n'est pas un groupe qui ne doit pas bénéficier automatiquement des services de l'internat. Des interventions que Finckenauer (1984) classe comme de nature sociale sont nécessaires en premier lieu, à savoir réduire leur aliénation face aux institutions, en regard de l'école et/ou du travail, de diminuer les influences des pairs délinquants qui les dominent, de leur rendre accessible des opportunités d'occuper des rôles sociaux légitimes (en référence au concept d'entrée en rôle d'adolescent), et sur le plan personnel, de valoriser leur estime de soi (en référence au concept de nature du fonctionnement psychologique).

Le délinquant à structure névrotique se caractérise par une délinquance qui est explosive, elle peut être numériquemment importante à certains moments et/ou grave, mais elle se résorbe par la suite. Ces adolescents délinquants se révèlent surtout menacés par le danger du désengagement interpersonnel; les liens avec autrui sont nettement appauvris; et l'entrée en rôle social semble assez fortement compromise. Ce désengagement s'appuie surtout, semble-t-il, sur la présence d'une ambivalence considérable qui marque plusieurs secteurs essentiels de leur vie. C'est la vision conflictualisée des autres et d'eux-mêmes qui alimenterait leur délinquance temporaire (en référence aux concepts de liens sociaux et de nature du fonctionnement psychologique). L'approche psychoéducative, qui a été développée au Québec (voir LeBlanc, 1983a, pour une évaluation d'un tel programme), apparaît celle qui répond le mieux aux besoins de ce type de jeunes délinquants.

Finalement, nous avons caractérisé le délinquant persistant grave, celui dont l'activité délictueuse dure, prolifère et s'aggrave pour inéluctablement se muer en criminalité adulte. Ils sont nettement coupés des instances sociales essentielles et ils se retrouvent dans une sorte de vacuum social; non seulement le fossé est-il creusé par rapport aux figures humaines significatives, mais il s'élargit par la prépondérance des allégeances aux modèles antisociaux. Dans le même sens, une accumulation prononcée de ce qui peut être appelé de la pathologie se fait jour dans certaines facettes essentielles de la personnalité; concept de soi négatif, 
méfiance, hostilité, irritation, sentiment d'être coupé des autres, ... Il est inévitable que ces jeunes se retrouveront devant le tribunal, dans les internats et, même, dans les internats de garde fermés. Il faut donc les reconnaître rapidement et intervenir massivement si nous voulons courir quelques chances de les resocialiser. Il est toutefois facile de faire mieux qu'actuellement, car $93 \%$ d'entre eux deviennent des criminels adultes. Les interventions doivent être précoces et massives; elles doivent s'attaquer à ce noyau central d'une personnalité égocentrique qui est déjà cristallisée (en référence au concept de nature du fonctionnement psychologique). Pour ce faire, quelques méthodes s'avèrent prometteuses : la modification du comportement, la clarification des valeurs qui met l'accent sur les rapports entre les idées, les attitudes et les actions et les programmes dérivés des théories de la personnalité criminelle (voir Finckenauer, 1984; et Mathias et al., 1984; pour quelques exemples de programmes qui offrent des perspectives intéressantes avec les jeunes délinquants persistants et, même, violents).

En somme, un véritable modèle différentiel d'intervention est possible, parce que les connaissances nous permettent maintenant de mieux distinguer parmi les jeunes délinquants et, ainsi, de mieux articuler les objectifs sociaux, protection de la société et aide aux jeunes, les besoins personnels des jeunes délinquants, rassemblés sous des types flexibles, et les politiques et stratégies d'intervention appropriées. Pour atteindre cet idéal de gestion clinique différentielle, il faut peut-être réduire la compétition entre les mesures, les organismes et les professions, mais il faut principalement établir et mettre en marché des politiques précises à cet égard. Politiques qui s'appuient sur les connaissances scientifiques (paradigme théorique et données empiriques) et les expériences pratiques (résultats des expériences et de leurs évaluations); politiques qui soutiennent un véritable modèle d'intervention différentiel face aux délinquances. Cette pensée et ce discours différentialiste sont actuellement rares en criminologie et inexistants chez nous malgré les possibilités offertes par les législations et la structure des services sociaux.

\section{VERS UNE CRIMINOLOGIE INTÉGRATIVE}

Cet article se voulait le reflet d'un cheminement à la poursuite de l'objectif de l'intégration. Intégration de quatre composantes : une connaissance spécifique du phénomène de la délinquance, la 
construction d'une théorie générale, la vérification empirique de celle-ci et l'identification des implications pour la pratique criminologique. L'objectif de l'intégration que nous proposaient Szabo et Wolfgang nous apparaît pertinent, nécessaire et réalisable comme en fait foi l'illustration proposée. Qu'en pensez-vous?

Ce n'était qu'une ébauche, l'œuvre reste à achever. Pour y arriver, il ne faudra pas se laisser divertir par des modes passagères, il conviendra plutôt de rappeler constamment les balises suivantes : la pierre angulaire de la criminologie c'est l'analyse de la conduite délinquante; les théories dites de régulation sont celles qui résistent le mieux aux épreuves du temps et des vérifications empiriques; la multidisciplinarité est une condition essentielle de la construction de la criminologie; et les implications pratiques doivent constituer la préoccupation centrale de toute réflexion criminologique.

\section{RÉFÉRENCES}

FINCKENAUER, J.O. (1984), Iuvenile Delinquency and Corrections: the Gap Between Theory and Practice, Don Mills, Academy Press.

FRÉCHETTE, M., M. LEBLANC (1979), La délinquance cachée à l'adolescence, Inadaptation juvénile, cahier I, Montréal, Groupe de recherche sur l'inadaptation juvénile.

FRÉCHETTE, M., M. LEBLANC (1980), Pour une pratique de la criminologie : configurations de conduites délinquantes et portraits de délinquants, Inadaptation juvénile, cahier V, Montréal, Groupe de recherche sur l'inadaptation juvénile.

FRÉCHETTE, M., M. LEBLANC (1985), Des délinquances : émergence et développement, Chicoutimi, Gaétan Morin.

IESSOR, R., T.D. GRAVES, R.C. HANSON, S.L. JESSOR (1968), Society, Personality and Deviant Behavior, New York, Holt, Rinehart, Winston.

JESSOR, R., S.L. JESSOR (1977), Problem Behavior and Psychological Development : a Longitudinal Study of Youth, New York, Academic Press. 
LEBLANC, M. (1971), «La réaction sociale à la déłinquance juvénile : une analyse stigmatique», Acta criminologica, 4 : 112-191.

LEBLANC, M. (1983a), Boscoville: la rééducation évaluée, Montréal, HMH.

LEBLANC, M. (1983b), «Une théorie intégrative de la conduite délinquante», Annales de Vaucresson, 20 : 1-33.

LEBLANC, M. (1985a), «De l'efficacité d'internats québécois», Revue canadienne de psycho-éducation.

LEBLANC, M. (1985b), «Inadaptation et réussite sociale : épidémiologie prospective», Apprentissage et socialisation, 8,3 :

LEBLANC, M., L. BIRON (1981), Vers une théorie intégrative de la conduite délinquante des garçons, Recherche : structure et dynamique de la conduite délinquante, Montréal, Groupe de recherche sur l'jnadaptation juvénile.

LEBLANC, M., R. TREMBLAY (1985), les Héritiers: une approche développementale et systémique à la compréhension de l'inadaptation juvénile, Montréal, Centre international de criminologie comparé.

LEBLANC, M., M. OUIMET (1985), Validation d'une théorie intégrative de la régulation de la conduite délinquante, Montréal, Centre international de criminologie comparée.

LEWIN, K. (1933), Principles of Topological Psychology, New York, McGraw Hill.

Loi sur les jeunes contrevenants, S.C. 1982, C. 110

Loi sur la protection de la jeunesse, L.R.Q. 1984

MARTIN, J.M., J.P. FITZPATRICK (1965), Delinquent Behavior: a Redefinition of the Problem, New York, Random House.

MATHIAS, R.A., P. DEMURO, R.S. ALLINSON (1984), Violent Juvenile Offenders : an Anthology, Washington, National council on crime and delinquency.

REISS, A.J. (1951), «Delinquency as a failure of personal and social controls», American Sociological Review, 16 : 196-207.

SZABO, D. (1968), Criminologie en action: bilan de la criminologie contemporaine dans ses grands domaines d'application, Montréal, Presses de l'Université de Montréal.

SZABO, D., M. LEBLANC, L. DESLAURIERS (1968), "Interprétations psycho-culturelles de l'inadaptation juvénile dans la société de masse contemporaine», Acta criminologica, I : 9-135.

TRÉPANIER, J., R. GAGNON (1984), la Déjudiciarisation à la cour du Bien-Être social de Montréal, Montréal, École de criminologie.

WALLER, I., D. WEILER (1984), la Prévention du crime par le développe. ment social, Document de base et de référence. Ottawa, Conseil canadien de développement social.

WEST, D.J. (1982), Delinquency : Roots, Careers and Prospects, London, Heinemann.

WOLFGANG, M.E., F. FERRACUTI (1967), The Subculture of Violence : Towards an Integrated Theory in Criminology, Beverly Hills, Sage. 


\section{APPENDICE}

\section{TABLEAU 2}

Chez les rarcons : intercorrélations ${ }^{1}$ et analvse de régression multiple des composantes de la théorie

\begin{tabular}{|c|c|c|c|c|c|}
\hline $\begin{array}{l}\text { Sexe Statut } \\
\text { social }\end{array}$ & Liens & Contrainte & $\begin{array}{l}\text { Entrée } \\
\text { en } \\
\text { rôle }\end{array}$ & $\begin{array}{l}\text { Fonction- } \\
\text { nement } \\
\text { psycholo- } \\
\text { gique }\end{array}$ & $\begin{array}{l}\text { Conduite } \\
\text { délin- } \\
\text { quante }\end{array}$ \\
\hline \multicolumn{6}{|l|}{ Sexe } \\
\hline Statut social & 23 & , 16 & ,07 &, 13 & , 10 \\
\hline Liens & & 67 &, 50 &, 56 & ,40 \\
\hline Contrainte & & & .66 &, 57 & ,67 \\
\hline \multicolumn{5}{|l|}{ Entrée en } & ,60 \\
\hline \multicolumn{6}{|l|}{$\begin{array}{l}\text { Fonction- } \\
\text { nement psy- } \\
\text { chologique }\end{array}$} \\
\hline \multicolumn{6}{|l|}{$\begin{array}{l}\text { Conduite } \\
\text { délinquante }\end{array}$} \\
\hline \multicolumn{6}{|c|}{$\begin{array}{l}\text { 1. Il s'agit de corrélations canoniques sauf en ce qui concerne les } \\
\text { variables sexe, statut social et conduite délinquante (corrélations multiples). }\end{array}$} \\
\hline \multicolumn{4}{|c|}{$\mathbf{R}$ multiple } & $\mathbf{R}^{2}$ & $\mathbf{r}$ \\
\hline \multicolumn{2}{|l|}{ Contrainte sociale } & \multicolumn{2}{|l|}{, 66648} & ,44420 & 66648 \\
\hline \multicolumn{2}{|l|}{ Entrée en rôle } & \multicolumn{2}{|l|}{, 70008} & 49011 & ,59915 \\
\hline \multicolumn{2}{|c|}{ Fonctionnement psychologique } & \multicolumn{2}{|l|}{, 72755} &, 52934 &, 55507 \\
\hline \multicolumn{2}{|l|}{ Liens sociaux } & \multicolumn{2}{|l|}{, 74183} &, 55031 & 40189 \\
\hline \multicolumn{2}{|l|}{ Statut social de famille } & ,74209 & &, 55070 & 10282 \\
\hline
\end{tabular}

TABLEAU 3

Chez les filles : intercorrelations ${ }^{1}$ et analyse de régression multiple des composantes de la théorie

\begin{tabular}{|c|c|c|c|c|c|}
\hline Sexe & $\begin{array}{l}\text { Statut Liens } \\
\text { social }\end{array}$ & Contrainte & $\begin{array}{l}\text { Entrée } \\
\text { en } \\
\text { rôle }\end{array}$ & $\begin{array}{l}\text { Fonction- } \\
\text { nement } \\
\text { psycholo- } \\
\text { gique }\end{array}$ & $\begin{array}{l}\text { Conduite } \\
\text { délin- } \\
\text { quante }\end{array}$ \\
\hline $\begin{array}{l}\text { Sexe } \\
\text { Statut social } \\
\text { Liens } \\
\text { Contrainte } \\
\text { Entrée en } \\
\text { rôle } \\
\text { Fonction- } \\
\text { nement psy- } \\
\text { chologique } \\
\text { Conduite } \\
\text { délinquante }\end{array}$ & 24 & , 12 & $\begin{array}{l}, 04 \\
, 56 \\
, 60\end{array}$ & $\begin{array}{l}, 14 \\
, 70 \\
, 59 \\
, 48\end{array}$ & $\begin{array}{l}, 05 \\
, 44 \\
, 59 \\
, 53 \\
, 52\end{array}$ \\
\hline
\end{tabular}

1. Il s'agit de corrélations canoniques sauf en ce qui concerne les variables sexe, statut social et conduite délinquante (corrélations multiples). 
Contrainte sociale

Entrée en rôle

R multiple

$\mathbf{R}^{2}$

r

, 58822

, 34600

,58822

, 63079

39790

, 65361

,42721

, 66191

, 43812

Liens sociaux

, 66196

, 43819

, 53427

, 51845

, 43776

, 04455

\section{SCHÉMA 4}

Intercorrélations chez les garcons

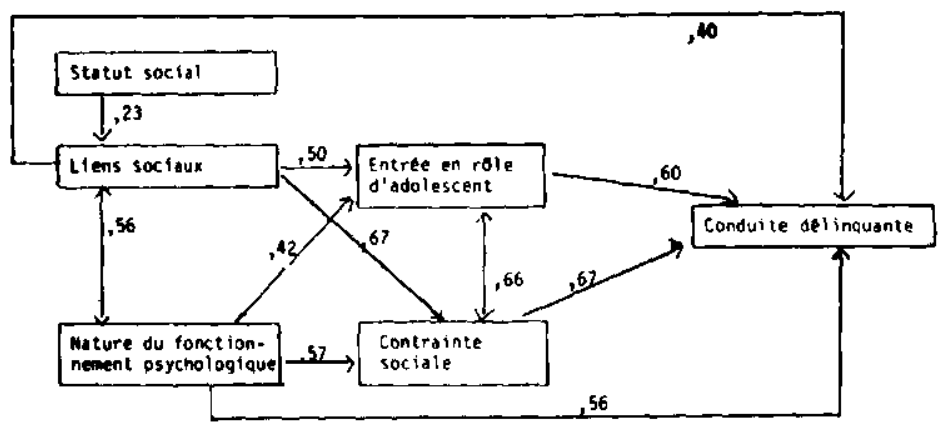

SCHÉMA 5

Intercorrélations chez les filles

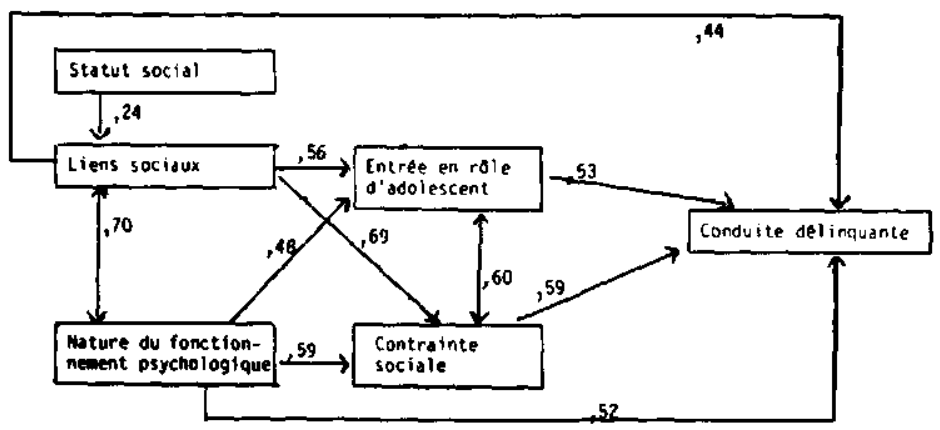




\begin{tabular}{|c|c|c|c|c|c|c|c|c|c|c|c|}
\hline Sexe & Statut & Lien 1 & $\begin{array}{l}\text { Con- } \\
\text { trainte } 1\end{array}$ & $\begin{array}{l}\text { Entrée } \\
\text { en } \\
\text { rôle } 1\end{array}$ & $\begin{array}{l}\text { Conduite } \\
\text { dél. } 1\end{array}$ & Lien 2 & $\begin{array}{l}\text { Con- } \\
\text { trainte } 2\end{array}$ & $\begin{array}{l}\text { Entrée } \\
\text { en } \\
\text { rôle } 2\end{array}$ & $\begin{array}{l}\text { Fonction- } \\
\text { nement } \\
\text { psycholo- } \\
\text { gique }\end{array}$ & $\begin{array}{l}\text { Conduite } \\
\text { dél. } 2\end{array}$ & \multirow{4}{*}{ 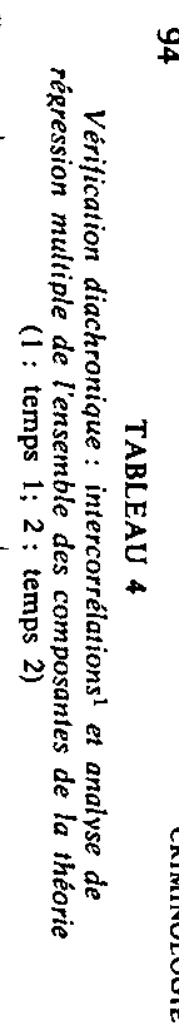 } \\
\hline \multirow[t]{3}{*}{$\begin{array}{l}\text { Sexe } \\
\text { Statut } \\
\text { Lien 1 } \\
\text { Contrainte 1 } \\
\text { Entrée en rôle 1 } \\
\text { Conduite délinquante } 1 \\
\text { Liens } 2 \\
\text { Contrainte } 2 \\
\text { Entrée en rôle } 2 \\
\text { Fonctionnement } \\
\text { psychologiqué } \\
\text { Conduite délinquante } 2 \\
\end{array}$} & .02 & $\begin{array}{l}, 11 \\
, 13\end{array}$ & $\begin{array}{l}, 29 \\
, 05 \\
, 64\end{array}$ & $\begin{array}{l}, 11 \\
, 07 \\
, 43 \\
, 50\end{array}$ & $\begin{array}{l}, 37 \\
.04 \\
, 37 \\
, 63 \\
, 52\end{array}$ & $\begin{array}{l}.09 \\
, 22 \\
, 58 \\
.48 \\
.36 \\
, 25\end{array}$ & $\begin{array}{l}, 34 \\
, 12 \\
, 46 \\
, 60 \\
, 39 \\
, 20 \\
, 67\end{array}$ & $\begin{array}{l}, 20 \\
, 06 \\
, 35 \\
.41 \\
.45 \\
.42 \\
.52 \\
.65\end{array}$ & $\begin{array}{l}, 45 \\
, 11 \\
.38 \\
, 34 \\
, 28 \\
.38 \\
, 62 \\
, 58 \\
, 44\end{array}$ & $\begin{array}{l}, 32 \\
.05 \\
, 21 \\
.38 \\
.27 \\
, 53 \\
, 40 \\
, 66 \\
, 60 \\
.54 \\
\end{array}$ & \\
\hline & \multicolumn{8}{|c|}{$\begin{array}{l}\text { 1. Il s'agit des corrélations canoniques sauf en ce qui concerne les } \\
\text { variables sexe, statut et conduite délinquante (corrélations multiples). }\end{array}$} & & & \\
\hline & $\begin{array}{l}\text { Contrai } \\
\text { Condui } \\
\text { Contrai } \\
\text { Entree } \\
\text { Sexe } \\
\text { Liens 2 } \\
\text { Entrée } \\
\text { Statut s } \\
\text { Liens 1 } \\
\text { Fonctio }\end{array}$ & $\begin{array}{l}\text { ne social } \\
\text { te délinq } \\
\text { nte socia } \\
\text { en rôle. } \\
\text { en rôle } 2 \\
\text { ocial } \\
\text { nnement }\end{array}$ & $\begin{array}{l}2 \\
\text { lante } 1 \\
\text { psychologio }\end{array}$ & 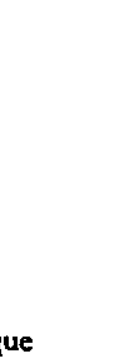 & $\begin{array}{l}\text { R multiple } \\
, 65948 \\
, 77475 \\
, 85961 \\
, 88182 \\
, 89133 \\
, 90150 \\
, 90257 \\
, 90330 \\
, 90371 \\
, 90394\end{array}$ & $\begin{array}{c}\mathrm{R}^{2} \\
, 43491 \\
, 60024 \\
.73892 \\
, 77761 \\
, 79446 \\
, 81270 \\
, 81464 \\
, 81595 \\
, 81669 \\
, 81711\end{array}$ & & $\begin{array}{c}r \\
65948 \\
52984 \\
37 \gamma 64 \\
26472 \\
32398 \\
40073 \\
59769 \\
04566 \\
21444 \\
54382\end{array}$ & & & \\
\hline
\end{tabular}




\section{SCHÉMA 6}

\section{Vérification diachronique : intercorrélation}

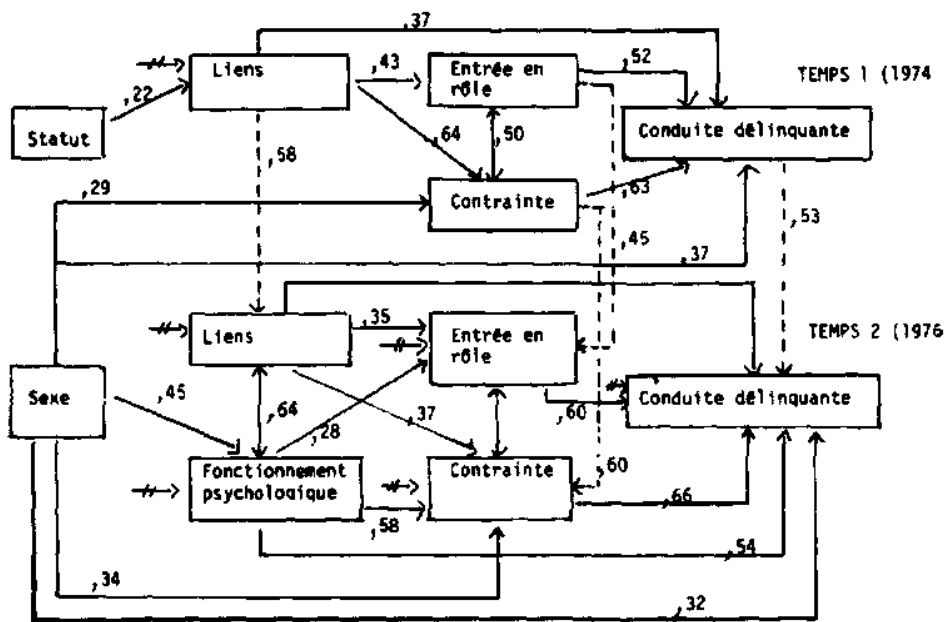

- Corralations synchroniques directes

--. Corrollations di achronlques directes

H Correlations importantes de toutes les variables du TI. 